\title{
Mathematical Modelling of an Atherosclerotic Blood Flow Through Double Stenosed Region with Application of Treatment
}

\author{
Kubugha Wilcox Bunonyo ${ }^{1,}$, , Emeka Amos ${ }^{2}$, Jason Biobaragha Goldie ${ }^{3}$ \\ ${ }^{1}$ Department of Mathematics and Statistics, Federal University Otuoke, Otuoke, Nigeria \\ ${ }^{2}$ Department of Mathematics, Rivers State University, Port Harcourt, Nigeria \\ ${ }^{3}$ Department of Biochemistry, Bayelsa State Polytechnic, Yenagoa, Nigeria
}

Email address:

wilcoxbk@fuotuoke.edu.ng (K. W. Bunonyo)

${ }^{*}$ Corresponding author

\section{To cite this article:}

Kubugha Wilcox Bunonyo, Emeka Amos, Jason Biobaragha Goldie. Mathematical Modelling of an Atherosclerotic Blood Flow Through Double Stenosed Region with Application of Treatment. International Journal of Applied Mathematics and Theoretical Physics.

Vol. 6, No. 2, 2020, pp. 19-25. doi: 10.11648/j.ijamtp.20200602.12

Received: May 31, 2020; Accepted: June 11, 2020; Published: June 20, 2020

\begin{abstract}
The aim of the present article is to model atherosclerotic blood flow through double stenosis in the arterial lumen with application of treatment subject to some boundary conditions in the channel. The research focused also on mathematical formulation of momentum equation governing the blood flow, driven by the oscillatory pressure gradient due to the contraction of muscles of the heart tissues and externally applied magnetic field. The nonlinear partial differential equation and geometries of the double stenosis are scaled using some quantities which made them dimensionless with some pertinent physical parameters such as the womersley number, the treatment parameter, Hartmann number, Darcy number at the region. The oscillatory perturbation method was adopted to further reduce the dimensionless equation to a Bessel differential equation of order zero. An exact solution of equation governing the flow is obtained with quantities coefficients. Mathematica codes were developed to investigate the influence of the pertinent parameters on the velocity profile, shear stress and volumetric flow rate of the first segment of stenosis $\delta_{1}$, while we considered the second stenosis $\delta_{2}$ segment fixed and present the results graphically. In conclusion, it is observed from the results and analysis that the pertinent parameters influenced the blood flow through a double stenosed region of an arterial lumen withholding the second stenosis fixed. These results could help in raising the awareness and the need for treatment of patients with double stenosis focusing on the most delicate.
\end{abstract}

Keywords: Treatment, Blood, Atherosclerosis, Flow, Darcy Number, Magnetic Field

\section{Introduction}

Atherosclerotic lesions of vessels are some of the most common vascular diseases and are classified as chronic dangerous pathologies. Atherosclerosis is a condition associated with formation of narrowing plaques on the vessel's walls. As a result, blood circulation, composition and properties of blood flow as well as the nutrition of organs and tissues do not run as usual. There are many reasons for this pathology: lack of physical activity, injury, and quality of food, age, family history and the presence of other diseases [1-4].

In recent time, medical science formulated several ways to perform diagnosis of stenosis such as ultrasound of the heart, magnetic - resonance angiography and computed tomography just to mention a few. Clinicians/medical practitioners performing the diagnostics often apply several procedures to investigate in order to have a complete picture of the disease dynamics. Mathematical formulation is a key and important method utilized in diagnostic and therapeutic surgery [5-6, 14]. Formulation of different mathematical models for different atherosclerotic cases allows and applying an individualistic approach to a particular case, forecasting and minimizing possible risks and making most appropriate effective treatment.

Creation of a mathematical model is difficult due to the complex internal structure of blood, nature of the blood flow, the structure of the vessel walls, and localization of the 
affected area of the circulatory system. In addition, limited amount of research has been done on mechanical properties of the formed elements in the blood flow. This is one of the reasons for the lack of consensus among researchers on necessity of considering non-Newtonian properties of the blood. Some scientists believe that the non-Newtonian properties should be considered only for calculations of small vessels (less than $0.5 \mathrm{~mm}$ [7]) when the value speed and tangential stresses are small so for relatively large elements of the circulatory system simulations carried out with Newtonian or power-law fluid models [8].

Since the beginning of the current century, scientists/mathematicians alike have undertaken attempts to investigate the blood flow as a Newtonian fluid using various models for the components of the circulatory system with a diameter of more than $1 \mathrm{~mm}$ [9]. Research [10] presents the results of an experiment, aimed at comparing the flow of Newtonian and viscoelastic liquids during the course of the flow in the branching element of a circulatory system. Glycerol solution was used as a Newtonian fluid while the diluted solution of polyacrylamide was used as a nonNewtonian. Three-dimensional channel of $1 \mathrm{~mm}$ diameter was used as a branching element of the circulatory system. The experiment was carried out for values of Reynolds number 498 to 951 and showed, that the stream lines for two fluids are different. A stagnant area formed in the channel in the case of a non-Newtonian fluid. This effect didn't observe in the case of Newtonian fluid. Studies [11-13] discuss modeling of the flow of blood in various kinds of elements of the circulatory system with pathologies (stenosis, aneurysms, and etc.) using Newtonian and non-Newtonian fluid models. A comparison of the results shows, that the representation of the blood as a nonNewtonian fluid allows to catch those effects, which arise in the flow of blood, but not reproduced by Newtonian fluid.

One of the most important characteristics of the blood flow in the circulatory systems is normal and shear stresses. Normal stresses arise where the blood flow meets a restriction or expansion of vessels, separation, as well as rotation of the flow. Relatively high values of normal stress may lead to the damage of the formed elements of the blood, leading to deformation of the vessels walls and formation of plaques. High normal stresses are the consequence of pressure increase in the blood and it is one of severe causative factor for heart attacks.

The main purpose of this work is to formulate a mathematical model of the flow of blood (a composition which contains protein macromolecules) in a stenosis affected elements of the circulatory system and discuss the influence of the pertinent parameters derived from the analytical solution of the governing model on the flow profile in the vicinity of the affected region of the blood circulatory system.

\section{Mathematical Formulation}

We consider blood to be a Newtonian, incompressible and viscous fluid, flowing through an atherosclerotic artery which we assumed to be a cylindrical channel $w^{*}\left(r^{*}, x^{*}\right)$ and porous.
The flow is caused by contraction of the heart and the oscillatory pressure gradient is in horizontal direction and the magnetic is applied perpendicularly to the flow direction. The azimuthaldirection of the flow is considered to be zero. Following Bunonyo et al [16] we present the equation governing the blood flow as:

$$
\rho \frac{\partial w^{*}}{\partial t^{*}}=-\frac{\partial p^{*}}{\partial x^{*}}+\frac{\mu}{r^{*}} \frac{\partial}{\partial r^{*}}\left(r^{*} \frac{\partial w^{*}}{\partial r^{*}}\right)-\frac{\mu}{k^{*}} w^{*}-\sigma B_{0}^{2} w^{*}
$$

Subject to the boundary condition:

$$
\left.\begin{array}{lll}
\frac{\partial w^{*}}{\partial r^{*}}=0 ; & \text { at } & r^{*}=0 \\
w^{*}=0 ; & \text { at } & r^{*}=R
\end{array}\right\}
$$

The region of atherosclerosis is assumed to be:

$$
\frac{r^{*}}{R_{0}}=\left\{\begin{array}{l}
1-\frac{\delta_{1}^{*}}{2 R_{0}}\left(1+\cos 2 \frac{\pi x^{*}}{\lambda}\right) \\
1-\frac{\delta_{2}^{*}}{2 R_{0}}\left(1+\cos 2 \frac{\pi x^{*}}{\lambda}\right)
\end{array}\right.
$$

where: $x=d_{0}+\frac{\lambda}{2}$

We introduce the following non-dimensional conditions in order solve equation (1) subject to the boundary condition in equation (2):

$$
\begin{aligned}
& x=\frac{x^{*}}{\lambda} ; t=t^{*} \omega ; D a=\frac{k^{*}}{R_{0}^{2}} ; w=\frac{2 \mu w^{*}}{q_{0} R_{0}^{2}} ; M=\frac{\sigma B_{0}^{2} R_{0}^{2}}{\mu} ; \\
& \left.\delta_{1}^{*}=\frac{2 \delta_{1} R_{0}}{R_{T}} ; \delta_{2}^{*}=\frac{2 \delta_{2} R_{0}}{R_{T}} ; r=\frac{r^{*}}{R_{0}} ; \frac{\partial P}{\partial x}=-q_{0} q(t), \alpha^{2}=\frac{R_{0}^{2} \omega}{v}\right\}
\end{aligned}
$$

Equations (1) \& (2) are transformed using equation (4), we obtain:

$$
\alpha^{2} \frac{\partial w}{\partial t}=2 q(t)+\frac{1}{r} \frac{\partial}{\partial r}\left(r \frac{\partial w}{\partial r}\right)-\frac{1}{D a} w-M^{2} w
$$

The geometry of the stenosis is now:

$$
h=\left\{\begin{array}{l}
1-\frac{\delta_{1}}{R_{T}}(1+\cos 2 \pi x) \\
1 \\
1-\frac{\delta_{2}}{R_{T}}(1+\cos 2 \pi x) \\
1
\end{array}\right.
$$

According to [15]: $q(t)=1+\gamma \cos (\omega t)$ 
Subject to the boundary condition:

$$
\begin{aligned}
& \left.\begin{array}{lll}
\frac{\partial w}{\partial r}=0 \quad \text { at } & r=0 \\
w=0 & \text { at } & r=h
\end{array}\right\} \\
& \alpha^{2} \frac{\partial w_{2}}{\partial t}=\frac{1}{r} \frac{\partial}{\partial r}\left(r \frac{\partial w_{2}}{\partial r}\right)-\left(\frac{1}{D a}+M^{2}\right) w_{2}+2 \gamma \cos (\omega t) \\
& \frac{1}{r} \frac{\partial}{\partial r}\left(r \frac{\partial w_{1}}{\partial r}\right)-\left(\frac{1}{D a}+M^{2}\right) w_{1}=-2
\end{aligned}
$$$$
\alpha^{2} \frac{\partial w_{2}}{\partial t}=2+2 \gamma \cos (\omega t)+\frac{1}{r} \frac{\partial}{\partial r}\left(r \frac{\partial\left(w_{1}+w_{2}\right)}{\partial r}\right)-\frac{1}{D a}\left(w_{1}+w_{2}\right)-M^{2}\left(w_{1}+w_{2}\right)
$$$$
\alpha^{2} \frac{\partial w}{\partial t}=2(1+\gamma \cos (\omega t))+\frac{1}{r} \frac{\partial}{\partial r}\left(r \frac{\partial w}{\partial r}\right)-\frac{1}{D a} w-M^{2} w
$$

Let $w=w_{1}(r)+w_{2}(r, t)$, then equation (8) is:

Applying transformation $\xi=\frac{r}{h}$ into equation (14) and equation (15) are reduced to the following:
Subject to the boundary condition:

$$
\left.\begin{array}{lll}
\frac{\partial w_{1}}{\partial r}=\frac{\partial w_{2}}{\partial r}=0 \quad \text { at } & r=0 \\
w_{1}=w_{2}=0 & \text { at } & r=h
\end{array}\right\}
$$

\section{Method of Solution}

In order to solve the dimensionless governing equation (6) subject to the boundary conditions in equation (9) as the heart pumps the blood through the circulatory channel, we assume the solution to be in the following form:

$$
\left.\begin{array}{l}
w_{1}=w_{1} e^{i \omega t} \\
w_{2}=w_{2} e^{i \omega t}
\end{array}\right\}
$$

Substitute equation (13) into the dimensionless equation (10) and equation (11), we obtain:

$$
\begin{gathered}
\frac{1}{r} \frac{\partial}{\partial r}\left(r \frac{\partial w_{2}}{\partial r}\right)-\beta_{2} w_{2}=-2 \gamma \cos (\omega t) e^{-i \omega t} \\
\frac{1}{r} \frac{\partial}{\partial r}\left(r \frac{\partial w_{1}}{\partial r}\right)-\beta_{1} w_{1}=-2
\end{gathered}
$$

where: $\beta_{1}=h_{1}^{2}\left(\frac{1}{D a}+M^{2}\right)$ and $\beta_{2}=h_{2}^{2}\left(\frac{1}{D a}+M^{2}+\alpha^{2} i \omega\right)$

Subject to the boundary condition:

$$
\left.\begin{array}{lll}
\frac{\partial w_{1}}{\partial r}=\frac{\partial w_{2}}{\partial r}=0 \quad \text { at } & r=0 \\
w_{1}=w_{2}=0 \quad \text { at } & r=h
\end{array}\right\}
$$

$$
\begin{gathered}
\frac{1}{\xi} \frac{\partial}{\partial \xi}\left(\xi \frac{\partial w_{2}}{\partial \xi}\right)-\beta_{2} w_{2}=-2 \gamma \cos (\omega t) e^{-i \omega t} \\
\frac{1}{\xi} \frac{\partial}{\partial \xi}\left(\xi \frac{\partial w_{1}}{\partial \xi}\right)-\beta_{1} w_{1}=-2
\end{gathered}
$$

Subject to the boundary condition:

$$
\left.\begin{array}{lll}
\frac{\partial w_{1}}{\partial \xi}=\frac{\partial w_{2}}{\partial \xi}=0 \quad \text { at } & \xi=0 \\
w_{1}=w_{2}=0 \quad \text { at } & \xi=1
\end{array}\right\}
$$

Solving the Bessel equation (18), we obtain:

$$
w_{1}(\xi)=A I_{0}\left(\sqrt{\beta_{1}} \xi\right)+B K_{0}\left(\sqrt{\beta_{1}} \xi\right)+\frac{2}{\beta_{1}}
$$

Where $I_{0}$ and $K_{0}$ are modified Bessel function of the first kind and second kind respectively.

$B=0$ as otherwise at $r=0$, then $w_{1}(\xi)$ is not finite. So, equation (16) is reduced to:

$$
w_{1}(\xi)=A I_{0}\left(\sqrt{\beta_{1}} \xi\right)+\frac{2}{\beta_{1}}
$$

Solving equation (12) subject to equation (19) we obtain the following:

$$
w_{1}(\xi)=\frac{2}{\beta_{1}}\left(1-\frac{I_{0}\left(\sqrt{\beta_{1}} \xi\right)}{I_{0}\left(\sqrt{\beta_{1}}\right)}\right)
$$

We solve equation as follows:

$$
w_{2}(\xi)=A_{2} I_{0}\left(\sqrt{\beta_{2}} \xi\right)+A_{3} K_{0}\left(\sqrt{\beta_{2}} \xi\right)+\frac{f}{\beta_{2}}
$$

where: $f=2 \gamma \cos (\omega t) e^{-i \omega t}$

Solving equation (23) subject to equation (19), we obtain 
the following:

$$
w_{2}(\xi)=\frac{f}{\beta_{2}}\left(1-\frac{I_{0}\left(\sqrt{\beta_{2}} \xi\right)}{I_{0}\left(\sqrt{\beta_{2}}\right)}\right)
$$

The general solution is the sum of equation (23) and equation (24):

$$
w=\left(\frac{2}{\beta_{1}}\left(1-\frac{I_{0}\left(\sqrt{\beta_{1}} \xi\right)}{I_{0}\left(\sqrt{\beta_{1}}\right)}\right)+\frac{f}{\beta_{2}}\left(1-\frac{I_{0}\left(\sqrt{\beta_{2}} \xi\right)}{I_{0}\left(\sqrt{\beta_{2}}\right)}\right)\right) e^{i \omega t}
$$

The volumetric flow rate is mathematically stated as:

$$
Q=2 \pi \int_{r=0}^{r=h} r w(r, t) d r
$$

Using the transformation, equation (26) is transformed as:

$$
Q=2 \pi\left(\frac{2}{\beta_{1}}\left(\frac{h_{1}^{2}}{\beta_{1}}-\frac{\sqrt{\beta_{1}} h_{1} I_{1}\left(\sqrt{\beta_{1}} \xi\right)}{\beta_{1} I_{0}\left(\sqrt{\beta_{1}}\right)}\right)+\frac{f}{\beta_{2}}\left(\frac{h_{2}^{2}}{\beta_{2}}-\frac{\sqrt{\beta_{2}} h_{2} I_{1}\left(\sqrt{\beta_{2}} \xi\right)}{\beta_{2} I_{0}\left(\sqrt{\beta_{2}}\right)}\right)\right) e^{i \omega t}
$$

The shear stress can be stated mathematically as follows:

$$
\tau_{w}=\left.\mu \frac{\partial w}{\partial r}\right|_{r=h}
$$

Applying the transformation, the shear stress is obtained as:

$$
\tau_{w}=\mu\left(\frac{2}{\beta_{1}}\left(\frac{\sqrt{\beta_{1}} I_{1}\left(\sqrt{\beta_{1}}\right)}{h_{1} I_{0}\left(\sqrt{\beta_{1}}\right)}\right)+\frac{f}{\beta_{2}}\left(\frac{\sqrt{\beta_{2}} I_{1}\left(\sqrt{\beta_{2}}\right)}{h_{2} I_{0}\left(\sqrt{\beta_{2}}\right)}\right)\right) e^{i \omega t}
$$

The impedance can be calculated using the pressure difference between the double stenosis in the lumen of an artery which can is stated mathematically as:

$$
K=\frac{\Delta P}{Q}
$$

Substituting the pressure values and the volumetric flow rate into equation (30), we obtained the following:

$$
K=\frac{f e^{-i \omega t}-2 e^{-i \omega t}}{2 \pi\left(\frac{2}{\beta_{1}}\left(\frac{h_{1}^{2}}{\beta_{1}}-\frac{\sqrt{\beta_{1}} h_{1} I_{1}\left(\sqrt{\beta_{1}} \xi\right)}{\beta_{1} I_{0}\left(\sqrt{\beta_{1}}\right)}\right)+\frac{f}{\beta_{2}}\left(\frac{h_{2}^{2}}{\beta_{2}}-\frac{\sqrt{\beta_{2}} h_{2} I_{1}\left(\sqrt{\beta_{2}} \xi\right)}{\beta_{2} I_{0}\left(\sqrt{\beta_{2}}\right)}\right)\right)}
$$

According to Einstein formula for the variable viscosity of blood taken to be

\section{Results}

In the previous section we have obtained analytical solutions for different flow characteristics of blood and heat transfer through an atherosclerotic artery with an externally applied magnetic field.

We used Mathematica 10 computer software to simulate the analytical results obtained for velocity profile, temperature profile, volumetric flow rate, wall shear stress and resistance to flow. We used the standard values $\alpha=2, R_{T}=0.2, \delta_{1}=2, \delta_{2}=2, M=3, D a=0.4, \omega=3, x=0.3$, in order to observe the significance of the parameters on the models as shown in the previous section in our discussion: Figures 1-12 present the velocity distribution for various

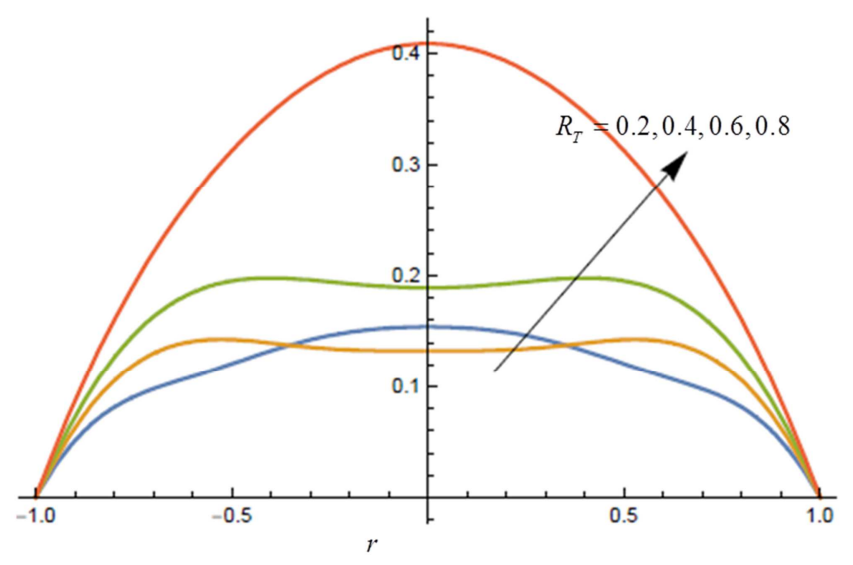

Figure 1. The influence of $R_{T}$ on blood flow, where other parameter values are taken as: $\alpha=2, \delta=2, M=3, D a=0.4, \omega=3, x=0.3$. flow parameters. 


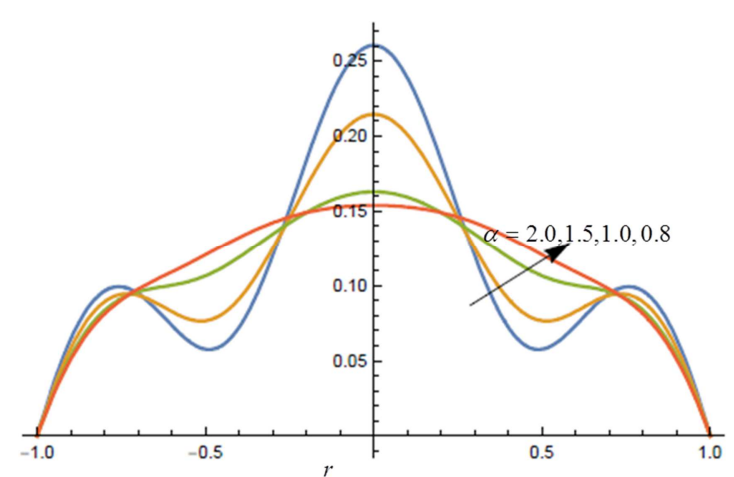

Figure 2. The influence of $\alpha$ on blood velocity, where other parameter values are taken as: $R_{T}=0.2, \delta=2, M=3, D a=0.4, \omega=3, x=0.3$.

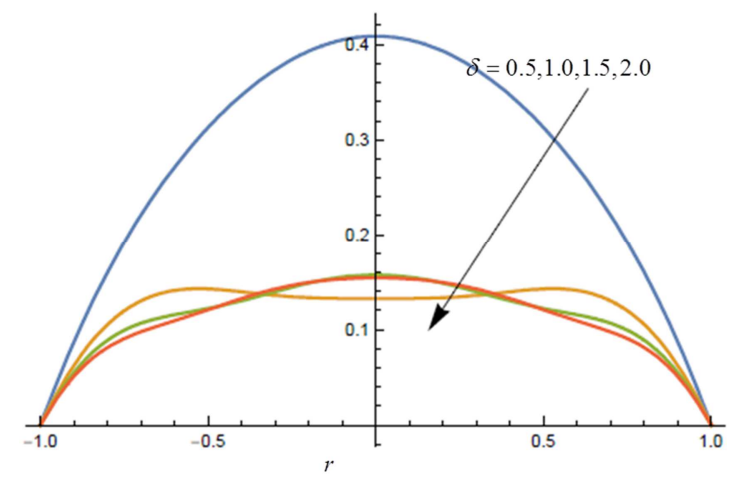

Figure 3. The influence of $\delta$ on blood velocity, where other parameter values are taken as: $R_{T}=0.2, \delta=2, M=3, D a=0.4, \omega=3, x=0.3$.

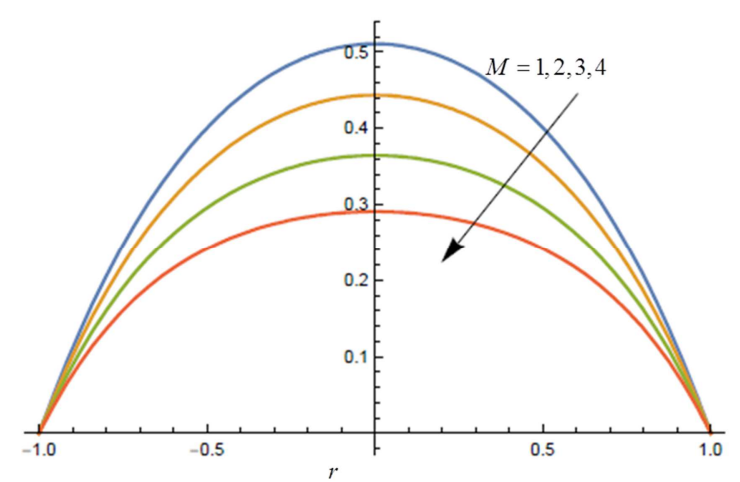

Figure 4. The influence of $M$ on blood velocity, where other parameter values are taken as: $R_{T}=0.2, \delta=2, \alpha=2, D a=0.4, \omega=3, x=0.3$.

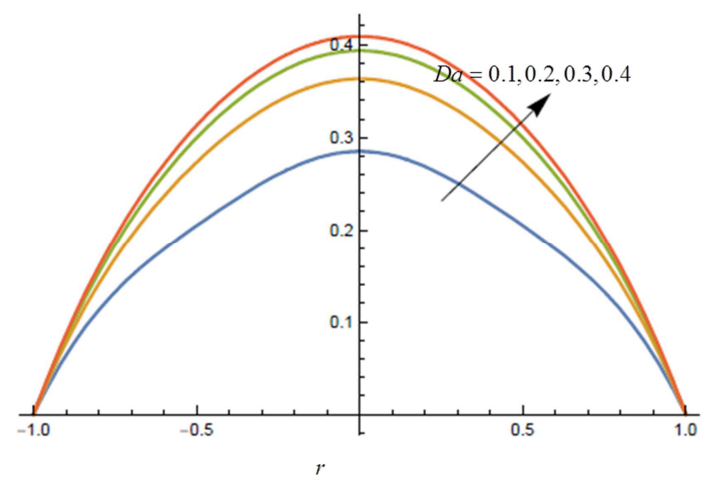

Figure 5. The influence of Da on blood velocity, where other parameter values are taken as: $R_{T}=0.2, \delta=0.5, M=3, \alpha=2, \omega=3, x=0.3$.

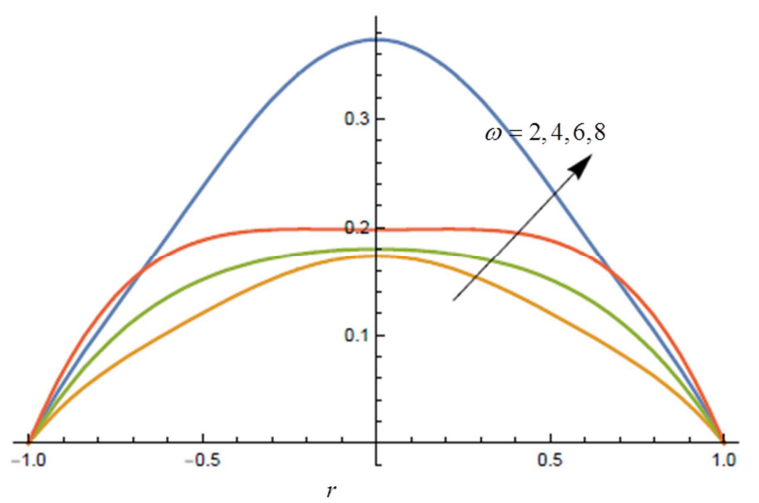

Figure 6. The influence of $\omega$ on blood velocity, where other parameter values are taken as: $R_{T}=0.2, \delta=0.5, M=3, D a=0.4, \alpha=2, x=0.3$.

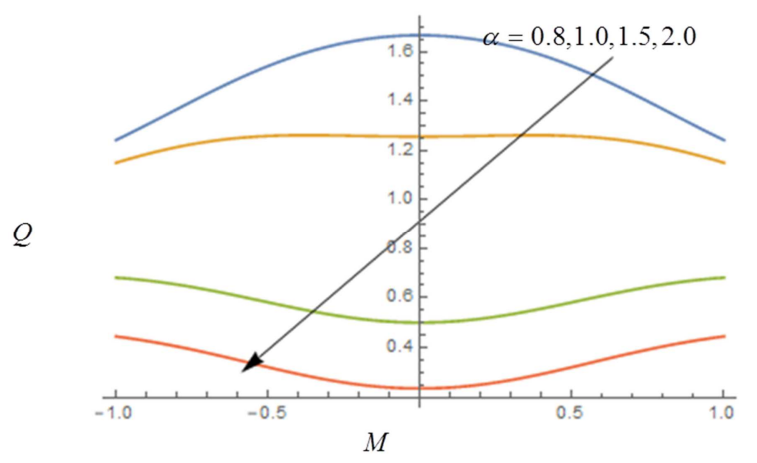

Figure 7. The influence of $\alpha$ on $Q$ against $M$, where other parameter values are taken as: $R_{T}=0.2, \delta=0.5, D a=0.4, x=0.3$.

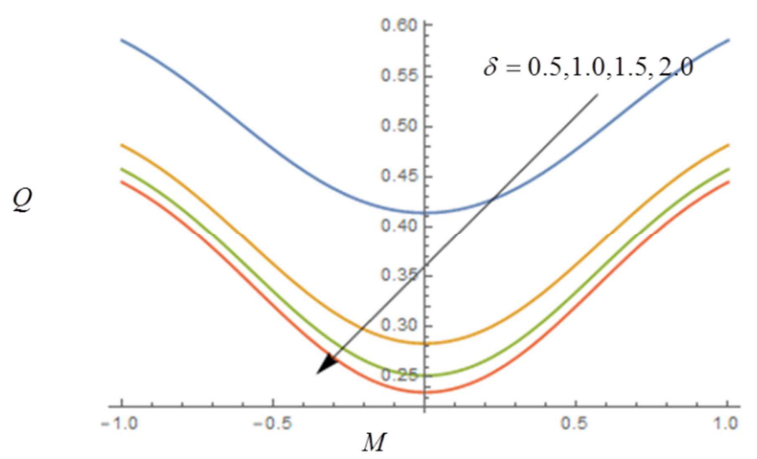

Figure 8. The influence of $\delta$ on $Q$ against $M$, where other parameter values are taken as: $R_{T}=0.2, D a=0.4, \alpha=2, x=0.3$.

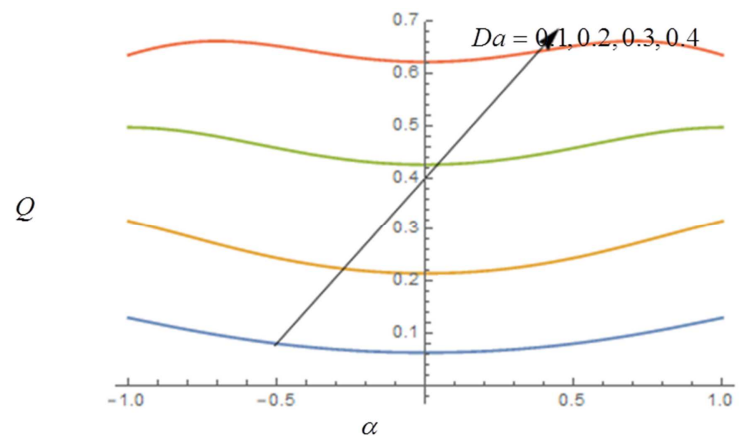

Figure 9. The influence of $D a$ on $Q$ against $\alpha$, where other parameter values are taken as: $R_{T}=0.2, \delta=0.5, M=3, x=0.3$. 


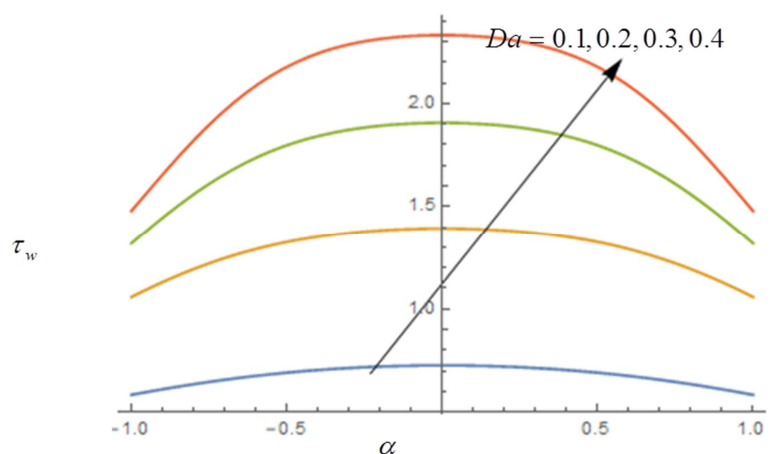

Figure 10. The influence of Da on $\tau_{w}$ against $\alpha$, where other parameter values are taken as: $R_{T}=0.2, \delta=0.5, M=3, x=0.3$.

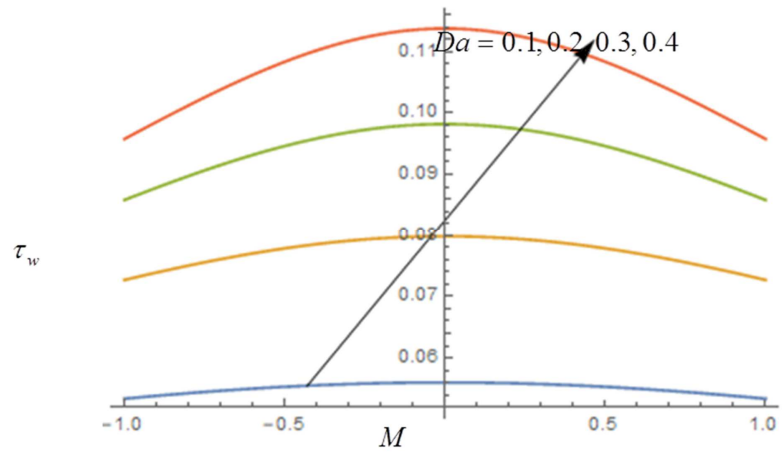

Figure 11. The influence of Da on $\tau_{w}$ against $M$, where other parameter values are taken as: $R_{T}=0.2, \delta=0.5, \alpha=2, x=0.3$.

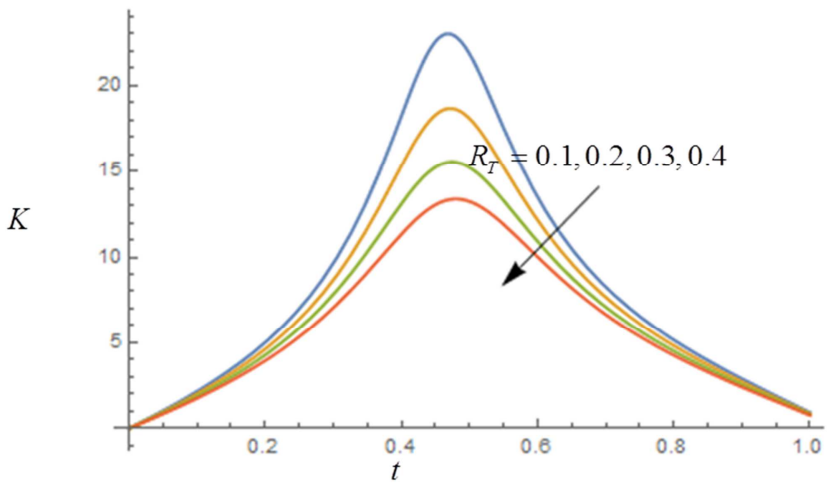

Figure 12. The influence of $R_{T}$ on $Q$ against $t$, where other parameter values are taken as: $\alpha=2, \delta_{1}=0.5, M=3, x=0.3$.

\section{Discussion}

In this section we are going to discuss the flow and heat transfer profiles graphically so as to extract the significance of information difficult to obtain by laboratory exercise and also to get a better understanding of the problem under investigation.

The influence of the pertinent parameters on the velocity and temperature profiles is discussed as: In Figure 1, it is noticed that increase in the treatment parameter gives rise to the increase in the blood velocity profile, the flow is seen to be maximum at the centre line where no stenosis before decelerating to zero at the point where the boundary layer is highest.

In Figure 2, this figure illustrates the influence of the womersley number on blood velocity profile; it is observed that as the velocity distribution decreases with increasing values of the parameter. The unique of this result is that, it clearly showed that the velocity at the centre line is maximum and relatively dropped to zero when the stenosis gets larger.

The impact of stenosis on the circulation of oxygenated blood cannot be overlooked in order to keep a watch on the cardiovascular challenge, in Figure 3, we noticed that the increase in stenosis as a result of excessive intake of LDL cholesterol, resulted to the inhibition of blood flow in the arterial channel even though the flow is maximum at the centre when there is no boundary layer.

The application of external magnetic field is of so much importance to blood circulation. If the magnetic field is applied to electrically conduction, moving fluid, it generates a force called the Lorentz force which opposes the blood flow as seen in Figure 4. The result for magnetic parameter can be implemented as a measure in the case of surgical procedure where blood flow needs to be monitored.

We noticed an influence of porosity on blood velocity distribution in Figure 5; in this figure it is observe that as porosity parameter values increases there is a corresponding increase in the blood velocity distribution. This result shows that even though the stenosis is a serious health channel with so much lip, there can be a prolonged life if well managed.

The oscillatory frequency parameter influence on the blood velocity distribution was simulated as seen in Figure 6. The figure show that increase in the oscillatory frequency increase the blood velocity distribution in the sense that the frequent one try the better the subsequent results.

In Figure 7, the figure illustrates the influence of womersley number on flow rate against the magnetic field intensity. It is clearly seen that increase in the parameter values increases the volumetric flow rate. It is also seen in Figure 8 that increase in $\delta$ values resulted to a corresponding decrease in volumetric flow rate due to excessive cholesterol intake.

Figures 8-9 depicts an influence of Darcy number on blood velocity distribution in the arterial channel; it is found that the porosity parameter values increase gives rise to volumetric flow rate. This flow rate is achieved because of the porosity on the stenosis. In Figures $10-11$, we notice an increase in shear stress against magnetic and womersley parameters while the porosity increased. Finally, the treatment parameter helps in decreasing the impedance of the blood flow in the arterial channel as the case in Figure 12.

\section{Conclusion}

An investigation was carried out on the topic: atherosclerotic blood flow through double stenosed region with treatment and magnetic effect. In our study we let second stenosis fixed and observe if the resulting pertinent 
parameters influence blood velocity distribution with the following findings:

The treatment parameter $R_{T}$ values influences blood velocity distribution in a stenosed artery with other pertinent parameter values are kept the same.

In a similar vein, the womersley parameter $\alpha$ values influenced blood velocity distribution in a stenosed artery with other pertinent parameter contributing to the flow.

The increase in the height of stenosis caused blood velocity to decrease, hence there is need to improve on the treatment mechanism, because the parameter influences it.

The importance of the magnetic field is seen from our investigation, we conclude that velocity profile inhibits blood velocity profile.

The porosity parameter also contributed to the improvement of blood velocity profile.

\section{Nomenclature}

$r^{*}$ : Dimensional perpendicular distance

$r$ : Dimensionless perpendicular distance

$x^{*}$ : Dimensional horizontal distance

$x$ : Dimensionless horizontal distance

$R_{0}$ : Radius of normal arterial segment

$R:$ Radius of abnormal arterial segment

$R e:$ Reynolds number

$\lambda, L_{0}$ : Length of arterial segment

$w^{*}$ : Dimensional blood velocity profile

$w$ : Dimensionless blood velocity profile

$\delta_{1,2}$ : Maximum height of stenosis

$R_{T}$ : Treatment parameter

$h$ : Boundary thickness

$d_{0}$ : Onset of stenosis

$t$ : Time parameter

$\mu$ : Blood dynamic viscosity

$\rho:$ Blood density

$\omega$ : Oscillatory frequency parameter

\section{References}

[1] Chang, R. W., Goodney, P. P., Baek, J. H., Nolan, B. W., Rzucidlo, E. M., \& Powell, R. J. (2008). Long-term results of combined common femoral endarterectomy and iliac stenting/stent grafting for occlusive disease. Journal of vascular surgery, 48 (2), 362-367.

[2] Dosluoglu, H. H., Lall, P., Cherr, G. S., Harris, L. M., \& Dryjski, M. L. (2010). Role of simple and complex hybrid revascularization procedures for symptomatic lower extremity occlusive disease. Journal of vascular surgery, 51 (6), 1425-1435.
[3] Adams, R. J., Albers, G., Alberts, M. J., Benavente, O., Furie, K., Goldstein, L. B.,... \& Katzan, I. (2008). Update to the AHA/ASA recommendations for the prevention of stroke in patients with stroke and transient ischemic attack. Stroke, 39 (5), 1647-1652.

[4] Brott, T. G., Hobson, R. W., Howard, G., Roubin, G. S., Clark, W. M., Brooks, W.,... \& Howard, V. J. (2010). Stenting versus endarterectomy for treatment of carotid-artery stenosis. New England Journal of Medicine, 363 (1), 11-23.

[5] Birchall, D., Zaman, A., Hacker, J., Davies, G., \& Mendelow, D. (2006). Analysis of haemodynamic disturbance in the atherosclerotic carotid artery using computational fluid dynamics. European radiology, 16 (5), 1074-1083.

[6] Sharafeev, A., Kutuzova, E., Tazyukov, F., Layek, G., \& Garifullin, F. Blood flow peculiarities in vessels bifurcation.

[7] Mandal, S. M., Mukhopadhyayy, S., \& Layek, G. C. Z. (2012). Pulsatile flow of shear-dependent fluid in a stenosed artery. Theoretical and Applied Mechanics, 39 (3), 209-231.

[8] Huh, H. K., Ha, H., \& Lee, S. J. (2015). Effect of nonNewtonian viscosity on the fluid-dynamic characteristics in stenotic vessels. Experiments in Fluids, 56 (8), 167.

[9] Cho, Y. I., \& Kensey, K. R. (1991). Effects of the nonNewtonian viscosity of blood on flows in a diseased arterial vessel. Part 1: Steady flows. Biorheology, 28 (3-4), 241-262.

[10] Moravec, S., \& Liepsch, D. (1983). Flow investigations in a model of a three-dimensional human artery with Newtonian and non-Newtonian fluids. Part I. Biorheology, 20 (6), 745-759.

[11] Perktold, K., Peter, R., \& Resch, M. (1989). Pulsatile nonNewtonian blood flow simulation through a bifurcation with an aneurysm. Biorheology, 26 (6), 1011-1030.

[12] Perktold, K. (1990). Non-Newtonian blood flow simulation and wall shear stress in an arterial bifurcation. In Biofluid Mechanics (pp. 471-477). Springer, Berlin, Heidelberg.

[13] Steffan, H., Brandstätter, W., Bachler, G., \& Pucher, R. (1990). Comparison of Newtonian and non-Newtonian blood flow in stenotic vessels using numerical simulation. In Biofluid Mechanics (pp. 479-485). Springer, Berlin, Heidelberg.

[14] Tazyukova, A. F., Tazyukov, F. K., Salman, H. D., Kutuzova, E. R., Kutuzov, A. G., \& Yushko, S. V. Blood flow through the circulatory system element affected by double stenotic lesions.

[15] Sharma, M. K., Sharma, P. R., \& Nasha, V. (2013). Pulsatile MHD Arterial Blood Flow in the Presence of Double Stenoses.

[16] Bunonyo, K. W., Israel-Cookey, C., \& Amos, E. (2018). Modeling of Blood Flow through Stenosed Artery with Heat in the Presence of Magnetic Field. Asian Research Journal of Mathematics, 1-14. 